Article

\title{
Cooperative and Independent Functions of the miR-23a 27a 24-2 Cluster in Bovine Adipocyte Adipogenesis
}

\author{
Yaning Wang ${ }^{1,+}$, Yingying Zhang ${ }^{2,+}$, Xiaotong Su ${ }^{1}$, Hongbao Wang ${ }^{1,3}$, Wucai Yang ${ }^{1,3}$ \\ and Linsen Zan ${ }^{1,3, *}$ \\ 1 College of Animal Science and Technology, Northwest A\&F University, Yangling 712100, China; \\ wangyn1992@outlook.com (Y.W.); xiaotongsu86@gmail.com (X.S.); \\ wanghongbao@nwsuaf.edu.cn (H.W.); yangwucai111@163.com (W.Y.) \\ 2 Animal Husbandry and Veterinary Research Institute, Shanghai Academy of Agricultural Sciences, \\ Shanghai 201106, China; zyy686868@163.com \\ 3 National Beef Cattle Improvement Center in China, Yangling 712100, China \\ * Correspondence: zanlinsen@163.com; Tel.: +86-029-8709-2484 \\ + These authors contributed equally to this work.
}

Received: 9 November 2018; Accepted: 4 December 2018; Published: 9 December 2018

\begin{abstract}
The miR-23a 27a 24-2 cluster is an important regulator in cell metabolism. However, the cooperative and independent functions of this cluster in bovine adipocyte adipogenesis have not been elucidated. In this study, we found that expression of the miR-23a 27a 24-2 cluster was induced during adipogenesis and this cluster acted as a negative regulator of adipogenesis. $m i R-27 a$ and $m i R-24-2$ were shown to inhibit adipogenesis by directly targeting glycerol-3-phosphate acyltransferase, mitochondrial (GPAM) and diacylglycerol O-acyltransferase 2 (DGAT2), both of which promoted adipogenesis. Meanwhile, $m i R-23 a$ and $m i R-24-2$ were shown to target decorin $(D C N)$, glucose-6-phosphate dehydrogenase $(G 6 P D)$, and lipoprotein lipase $(L P L)$, all of which repressed adipogenesis in this study. Thus, the $m i R-23 a \sim 27 a \sim 24-2$ cluster exhibits a non-canonical regulatory role in bovine adipocyte adipogenesis. To determine how the miR-23a 27a 24-2 cluster inhibits adipogenesis while targeting anti-adipogenic genes, we identified another target gene, fibroblast growth factor 11 (FGF11), a positive regulator of adipogenesis, that was commonly targeted by the entire $m i R-23 a \sim 27 a \sim 24-2$ cluster. Our findings suggest that the miR-23a 27a 24-2 cluster fine-tunes the regulation of adipogenesis by targeting two types of genes with pro- or anti-adipogenic effects. This balanced regulatory role of $m i R-23 a \sim 27 a \sim 24-2$ cluster finally repressed adipogenesis.
\end{abstract}

Keywords: $m i R-23 a \sim 27 a \sim 24-2$ cluster; bovine; adipocyte; adipogenesis

\section{Introduction}

Fat deposition is highly correlated with beef carcass grading and characteristics that contribute to meat quality such as juiciness, tenderness, and flavor. Therefore, it is essential to advance our understanding of the molecular mechanisms that affect fat deposition so that genomic selection can be used to produce high-grade beef with consistent quality. Adipose tissue is a remarkably complex organ that is involved in multiple physiological and pathological processes [1]. Adipogenesis of progenitor cells into mature adipocytes is tightly controlled by various factors [2,3]. Among these factors, miRNAs have been reported to have a close relationship with fat deposits and meat quality [4,5]. However, the exact roles of many miRNAs in adipogenesis have not yet been determined.

Mature miRNAs are small (approximately $22 \mathrm{nt}$ ), single-stranded non-cording RNAs that mainly repress gene expression at the post-transcriptional level by binding to target mRNAs [6]. 
During adipose tissue development and other biological processes, miRNAs mainly function individually or as clusters [7,8]. Generally, clustered miRNAs are expressed from the same transcript [9], and the host gene encoding these miRNAs possesses an intact gene structure [10]. The primary miRNA transcripts also contain a cap structure and a poly A tail like protein coding genes [10]. A previous study found that $37 \%$ of the known human miRNAs are located in clusters, and most of the clusters show a high degree of evolutionary conservation [11]. Usually, a miRNA cluster contains several to dozens of miRNAs, and the clustered miRNAs have similar but also individual functions [12].

The $m i R-23 a \sim 27 a \sim 24-2$ cluster, which is expressed from a single primary transcript, is involved in various physiological and pathological processes [12]. However, the expression patterns and functions of the miRNAs in this cluster are not always the same and are even opposite depending on the cell type and biological process [12]. Studies have shown that the miR-23a 27a 24-2 cluster regulates endothelial cell apoptosis [13], osteoblast differentiation [14,15], neuronal apoptosis [16], and erythropoiesis [17]. Recent studies in humans have also indicated that the miR-23a 27a 24-2 cluster may also significantly affect adipogenesis because many of their predicted target genes are involved in multiple signaling pathways related to lipid metabolism [12]. During the differentiation of bovine adipocyte progenitor cells and 3T3-L1 cells, miR-23a serves as a negative regulator [18,19]. miR-27a represses 3T3-L1 preadipocyte differentiation by directly targeting peroxisome proliferator activated receptor gamma $(P P A R \gamma)$ [20]. miR-27a is also involved in brown adipogenesis [21], porcine preadipocyte differentiation [22], and high-fat diet induced insulin resistance [23]. Based on these reports, we hypothesize that the functions of the miRNAs in this cluster are not exactly the same and that they have the crucial cooperative regulatory roles in the process of bovine preadipocyte differentiation. Therefore, it is essential to elucidate the molecular mechanisms by which the miR-23a 27a 24-2 cluster affects fat deposition so that this knowledge can be applied to genomic selection to produce high-grade beef.

In the process of preadipocyte differentiation, fatty acids, and lipid synthesis pathways, such as oxidative phosphorylation, pentose phosphate pathway, and triacylglycerol (TAG) synthesis pathway, play essential roles [24-26]. In these cellular bioprocesses, adenosine triphosphate synthase peripheral stalk-membrane subunit b (ATP5F1) is important for oxidative phosphorylation [25], G6PD is essential for pentose phosphate pathway [27], and GPAM, DGAT2, DCN, and LPL are the key regulators for TAG synthesis [28-30]. Although it is much clearer how these genes affect adipogenesis, the interactions between these pathways and miRNA networks are largely unknown.

The aim of this study was to identify the mechanisms of the miR-23a 27a 24-2 cluster in regulating bovine adipocyte adipogenesis. Through computational analysis, we found that there might be post-transcriptional regulations between the miRNAs in the miR-23a 27a 24-2 cluster and genes related to lipid synthesis. Consequently, we hypothesize that the miR-23a 27a 24-2 cluster might influence adipogenesis through interactions with lipid synthesis-related pathways. It is intriguing that the newly identified cooperative and independent regulatory roles of the miRNAs in this cluster will help inform future studies of preadipocyte differentiation, which can be applied to enhance fat deposition and meat quality of beef.

\section{Results}

\subsection{Isolation and Adipogenic Differentiation of Bovine Preadipocytes}

To establish a system to study the effects of miRNAs on the adipogenic differentiation of bovine preadipocytes, the adipogenic potential of preadipocytes isolated from perirenal adipose tissue was evaluated. As shown in Figure 1A, $24 \mathrm{~h}$ after isolation, cells started to adhere to the dishes and began to elongate. Seventy-two hours later, cells had grown to $70 \%$ confluence and were spindle-shaped (Figure 1A). When cells were 100\% confluent, adipogenic differentiation of the preadipocytes was induced. The shape of the preadipocytes gradually changed from shuttle-shaped to oblate during adipogenesis. On the 15th day of differentiation, almost all cells were induced to mature adipocytes 
with strong adipogenic potential. The expression levels of the adipogenic marker genes PPARG, CCAAT enhancer binding protein alpha (CEBPA), and sterol regulatory element binding transcription factor 1 (SREBP1) were all obviously induced during differentiation (Figure 1B). These results indicate that the isolated bovine preadipocytes were suitable for subsequent experiments.

A

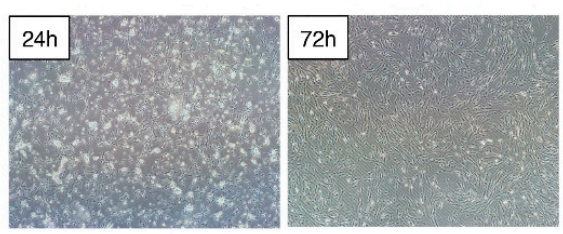

$\mathrm{B}$

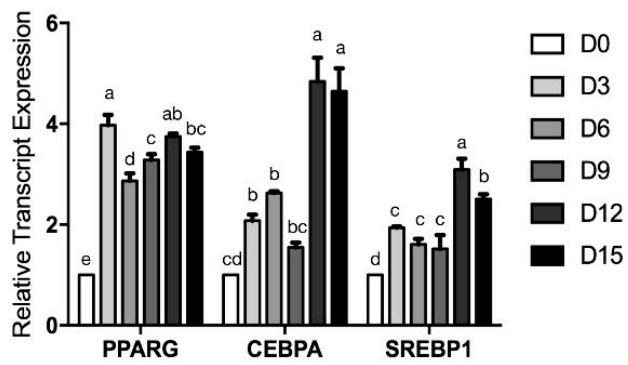

Figure 1. Isolation and adipogenic differentiation of bovine preadipocytes. (A) Initial isolation and cell culture of bovine primary preadipocytes at 24 and $72 \mathrm{~h}$ (magnification: $40 \times$ ). (B) Time course of $P P A R G, C E B P A$, and SREBP1 expression during preadipocyte differentiation. Error bars represent SEM.

Lowercases represent $p<0.05$. Uppercases represent $p<0.01$.

\subsection{The miR-23a 27a 24-2 Cluster Represses Preadipocyte Differentiation}

To reveal the relationships between the miR-23a 27a 24-2 cluster and adipogenesis, the time course of $m i R-23 a, m i R-27 a$, and $m i R-24-2$ expression from day 0 (D0) to D15 of differentiation were analyzed by performing quantitative real time PCR (qRT-PCR) (Figure 2A-C). miR-23a expression was induced starting on D3 and then remained stable (Figure 2A). miR-24-2 expression was also induced from $\mathrm{D} 3$ to $\mathrm{D} 9$ and then the expression level gradually decreased (Figure $2 \mathrm{C}$ ).

A

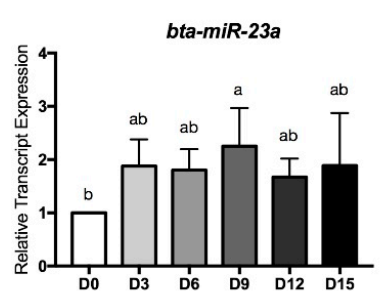

B

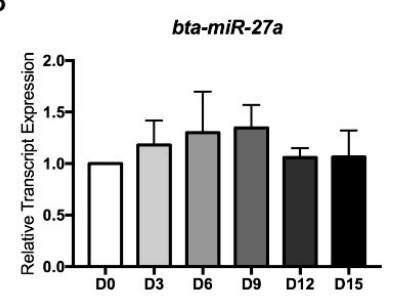

C

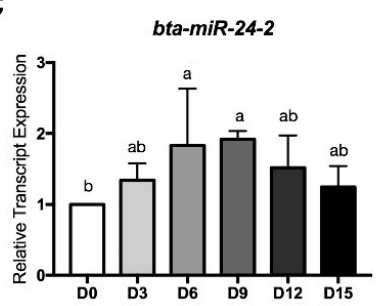

D
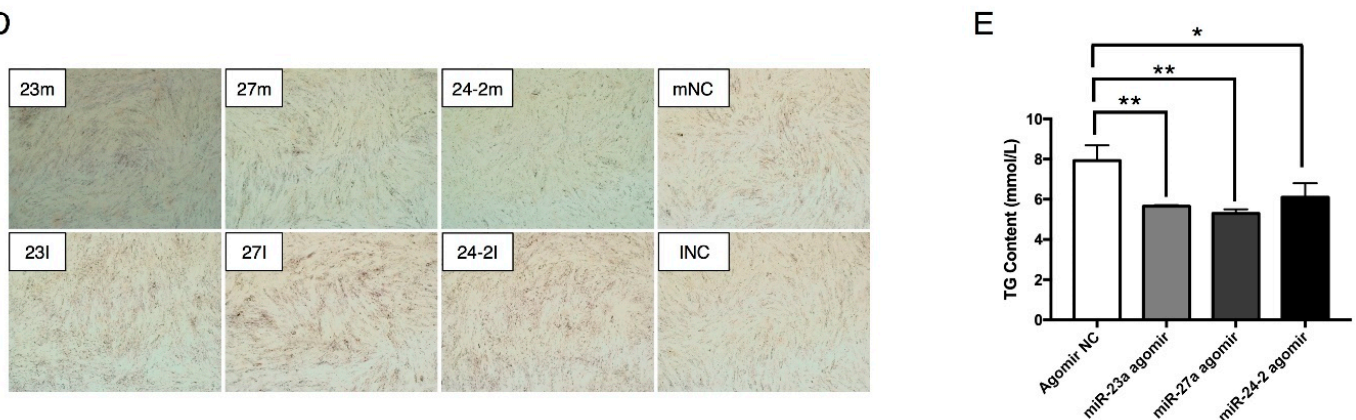

Figure 2. The miR-23a 27a 24-2 cluster represses preadipocyte differentiation. (A-C) Time course of $m i R-23 a, m i R-27 a$, and $m i R-24-2$ expression during preadipocyte differentiation. (D) Oil red O staining of lipids in differentiated adipocytes transfected with $m i R-23 a$ agomir $(23 \mathrm{~m})$ / antagomir (23I), miR-27a agomir (27m)/antagomir (27I), miR-24-2 agomir (24-2m)/antagomir (24-2I), negative control (NC) for agomir (mNC) and NC for antagomir (INC, magnification: 40×). (E) ELISA analysis of TG content in adipocytes transfected with Agomir NC, miR-23a agomir, miR-27a agomir and miR-24-2 agomir. Error bars represent SEM. Lowercase represents $p<0.05$. Uppercase represents $p<0.01$. " $* *$ " represents $p<0.05 ;{ }^{\prime * * * \prime \prime}$ represents $p<0.01$. 
To investigate the effects of $m i R-23 a, m i R-27 a$, and miR-24-2 on preadipocyte differentiation, $m i R-23 a$ agomir/antagomir, miR-27a agomir/antagomir, and miR-24-2 agomir/antagomir were transfected individually into proliferating preadipocytes. When the cells were $100 \%$ confluent, the preadipocytes were induced to differentiation. The lipid droplets were observed by staining the cells with Oil Red $\mathrm{O}$ at D5 after adipogenic induction. As shown in Figure 2D, the $m i R-23 a, m i R-27 a$, and miR-24-2 agomir repressed lipid droplet formation. In contrast, the antagomir promoted preadipocyte differentiation and increased lipid accumulation. Meanwhile, the total cytoplasm triglyceride (TG) content was also significantly repressed by the miRNA agomir (Figure 2E). These results indicate that the miR-23a 27a 24-2 cluster is a negative regulator of bovine preadipocyte differentiation.

2.3. The miR-23a 27a 24-2 Cluster Negatively Regulates the Expression of ATP5F1, DCN, GPAM, DGAT2, $G 6 P D$, and $L P L$

By using TargetScan software (Release: 7.2: http://www.targetscan.org/vert_72/) and miRanda (http://www.microrna.org/microrna/home.do), six genes were identified as potential targets of the miR-23a 27a 24-2 cluster: ATP5F1, DCN for miR-23a, GPAM for miR-27a, and DGAT2, G6PD, and $L P L$ for $m i R-24-2$. First, the expression patterns of the six genes were analyzed using qRT-PCR. As shown in Figure 3A-C, apart from DGAT2, whose expression was continuously induced from D0 to D12 and decreased at D15, gene expression levels of the other five genes first increased at D3 of differentiation, and then decreased at D6 to D9 before increasing again. These expression patterns are opposite to those of $m i R-23 a, m i R-27 a$, and $m i R-24-2$, suggesting that these six genes might be regulated by the miR-23a 27a 24-2 cluster. To verify this, the expression levels of the six genes were analyzed with adipocytes transfected with miRNA agomir or antagomir. Results showed that the miR-23a agomir strongly repressed ATP5F1 and DCN expression (Figure 3D). In contrast, the miR-23a antagomir obviously increased the expression of these genes from $36 \mathrm{~h}$ to 8 days post transfection (Figure 3G). The expression of GPAM was also inhibited by the $m i R-27 a$ agomir and induced by the miR-27a antagomir (Figure 3E,H). The miR-24-2 agomir significantly reduced the expression of DGAT2 and $L P L$, but had no effect on G6PD expression at $36 \mathrm{~h}$ post transfection (Figure 3F). By contrast, inhibition of miR-24-2 by its antagomir strongly increased DGAT2, G6PD, and LPL expression; this increase was especially pronounced for $G 6 P D$ at $36 \mathrm{~h}$ post transfection (Figure $3 \mathrm{I})$. These results strongly suggest that ATP5F1, DCN, GPAM, DGAT2, G6PD, and LPL are targets of the miR-23a 27a 24-2 cluster.

A

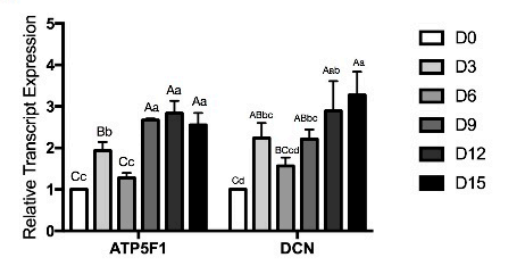

D

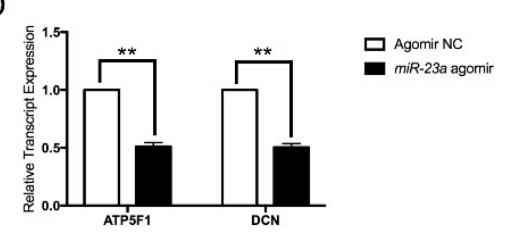

B

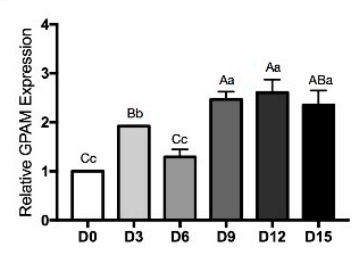

E

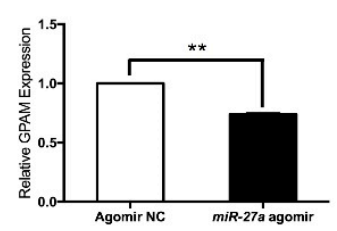

C

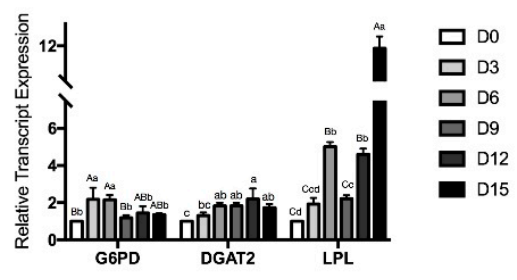

F

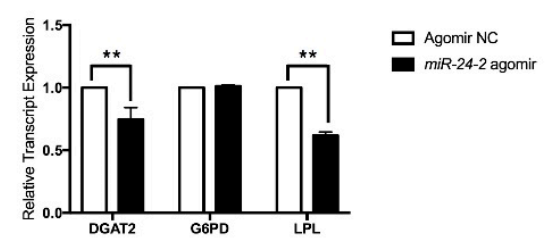

Figure 3. Cont. 
G

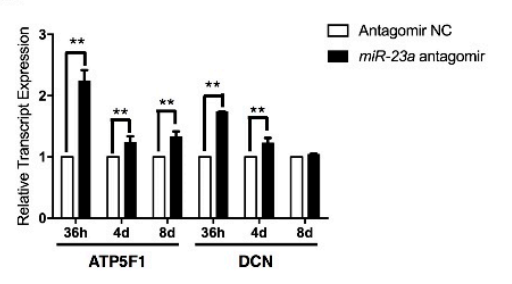

$\mathrm{H}$

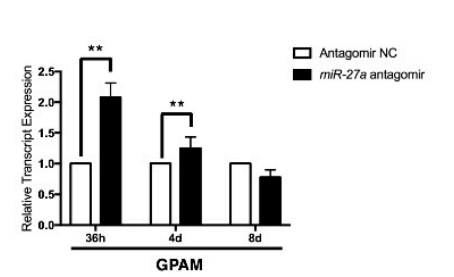

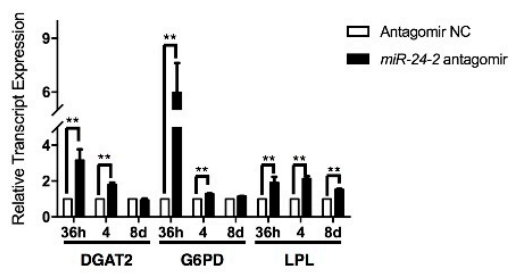

Figure 3. The miR-23a 27a 24-2 cluster negatively regulates the expression of ATP5F1, DCN, GPAM, DGAT2, G6PD, and LPL. (A-C) Time course of ATP5F1, DCN, GPAM, DGAT2, G6PD, and LPL expression during preadipocyte differentiation. (D-F) mRNA expression of ATP5F1, DCN, GPAM, DGAT2, G6PD, and LPL in preadipocytes individually transfected with $m i R-23 a$ agomir, $m i R-27 a$ agomir, and $m i R-24-2$ agomir, followed by adipogenic differentiation at $36 \mathrm{~h}$. (G-I) Transfection of miR-23a antagomir, $m i R-27 a$ antagomir, and $m i R-24-2$ antagomir in preadipocytes, followed by adipogenic differentiation increased ATP5F1, DCN, GPAM, DGAT2, G6PD, and LPL expression from $36 \mathrm{~h}$ to 8 days. Error bars represent SEM. Lowercase represents $p<0.05$. Uppercase represents $p<0.01$. " “*** represents $p<0.01$.

2.4. The miR-23a 27a 24-2 Cluster Directly Targets Two Types of Genes: GPAM and DGAT2 with Pro-Adipogenic Effects and DCN, G6PD, and LPL with Anti-Adipogenic Effects

To investigate whether ATP5F1, DCN, GPAM, DGAT2, G6PD, and LPL were direct targets of the miR-23a 27a 24-2 cluster, in vitro luciferase activity assays were performed. Wild type or mutant $3^{\prime}$ UTR luciferase reporter vectors were co-transfected with miRNA agomir or the NC agomir in 293T cells. Results showed that miR-23a agomir significantly reduced the luciferase activities of the ATP5F1 and DCN 3'UTR reporter vectors (Figure 4A,B), and the miR-27a agomir repressed the luciferase activity of the GPAM reporter vector (Figure 4C). The miR-24-2 agomir clearly decreased the luciferase activity of the DGAT2, G6PD, and LPL reporter vectors (Figure $4 \mathrm{D}-\mathrm{F}$ ). The results from these luciferase activity assays confirmed that ATP5F1, DCN, GPAM, DGAT2, G6PD, and LPL are direct target genes of the miR-23a 27a 24-2 cluster.

A

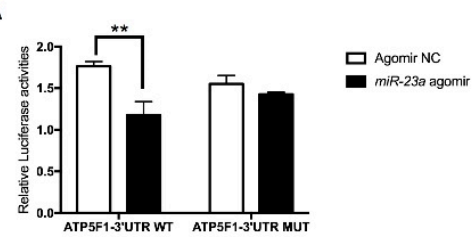

D

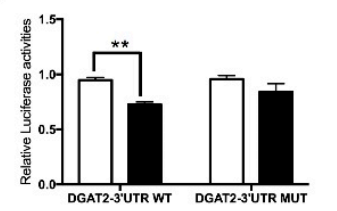

B

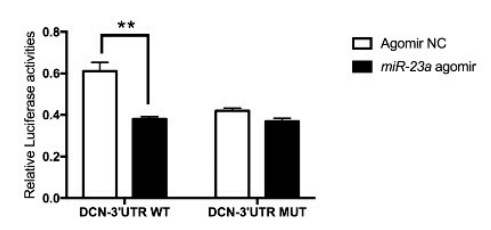

E

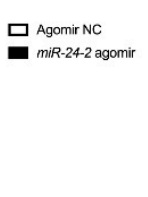

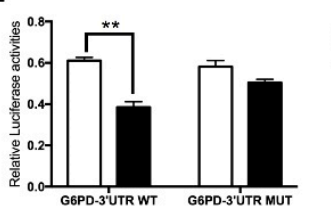

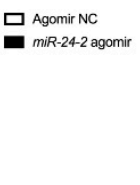

C

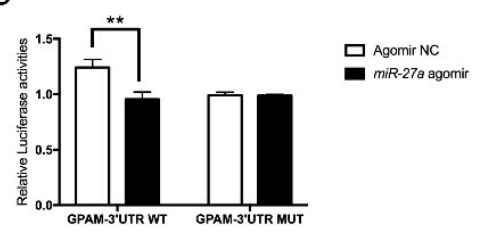

F

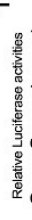

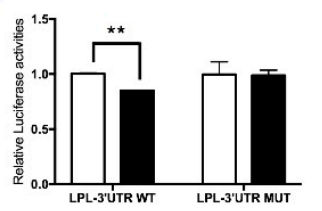

ㅁ Agomir NC miR-24-2 agomir

Figure 4. The miR-23a 27a 24-2 cluster directly targets ATP5F1, DCN, GPAM, DGAT2, G6PD, and LPL. $(\mathbf{A}, \mathbf{B})$ Luciferase activity assay of ATP5F1-3'UTR and DCN-3'UTR targeted by miR-23a. (C) Luciferase activity assay of GPAM-3'UTR targeted by miR-27a. (D-F) Luciferase activity assay of DGAT2-3'UTR, G6PD-3'UTR, and LPL-3'UTR targeted by miR-24-2. Error bars represent SEM. “**” represents $p<0.01$.

Most of the present published literatures reported that the effects of miRNA and their targets were opposite $[14,31]$. To investigate the roles of the six target genes on preadipocyte differentiation, interference assays were performed using siRNA. Due to the difficulty of designing an siRNA targeting ATP5F1 mRNA, ATP5F1 was not included in this study. Surprisingly, transfection of cells with siRNA targeting DCN or LPL significantly increased lipid content, as visualized by bright field microscopy (Figure 5A) and Oil Red O staining (Figure 5B). ELISA analysis of TG content also showed that inhibition of LPL expression increased adipocyte TG content (Figure 5C). Similarly, interference of 
G6PD obviously promoted adipogenesis, but inhibition of DGAT2 reduced lipid content (Figure 5B). As it was not easy to recognize how GPAM affected adipogenesis using Oil Red O staining (Figure 5B), and the results of ELISA analysis showed that the interference of GPAM expression reduced adipocyte TG content. These results suggest that the five targets were sorted into two groups: GPAM and DGAT2 with pro-adipogenic effects, and $D C N, G 6 P D$, and $L P L$ with anti-adipogenic effects. However, it is not clear how the miRNA cluster acts as a negative regulator of adipogenesis when most of its target genes are anti-adipogenic.

A

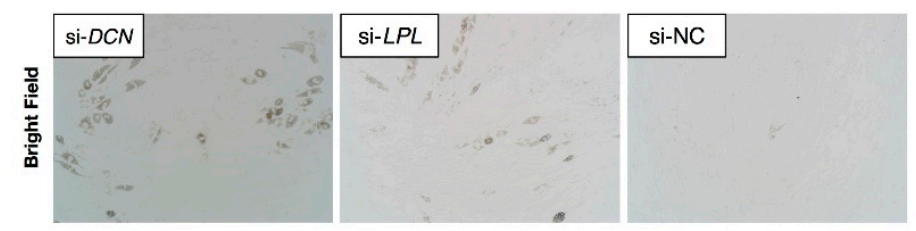

B

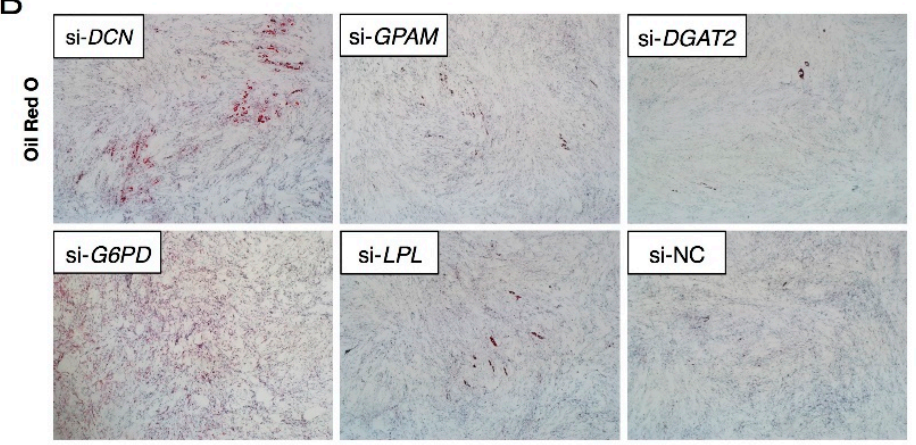

\section{C}

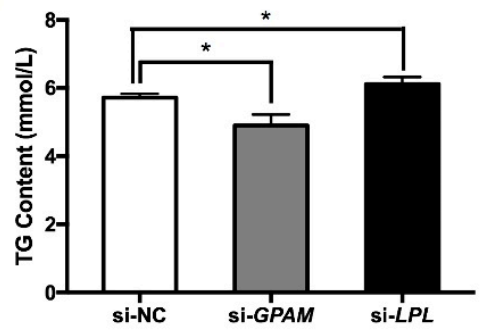

Figure 5. Interference effects of DCN, GPAM, DGAT2, G6PD, and $L P L$ on adipocyte adipogenesis. (A) Bright field microscopy of lipid droplets in $D C N$ - and $L P L$-interfered preadipocytes followed by differentiation at day 5 (magnification: $100 \times$ ). As there were no obvious lipid droplets observed in the bright field microscopy when interfering GPAM, DGAT2, and G6PD, the data was not shown. (B-C) Oil Red O staining of lipids (magnification: $40 \times$ ) and ELISA analysis of TG content showed that $D C N, G 6 P D$, and LPL were strongly anti-adipogenic while GPAM and DGAT2 were pro-adipogenic in adipogenesis. Error bars represent SEM. "**" represents $p<0.05$.

2.5. Hypothesis: There are Other Pro-Adipogenic Target Genes that Contribute to the miR-23a 27a 24-2 Cluster-Regulated Adipogenesis

As shown in Figure 6A,B, miR-23a 27a 24-2 potentially regulated adipogenesis by regulating two types of targets: genes with anti-adipogenic effects (solid blue lines) and pro-adipogenic effects (dotted green lines). Because we verified that the miR-23a 27a 24-2 cluster inhibits preadipocyte differentiation, the predominant role of the target genes should theoretically be pro-adipogenic. However, our results indicate that $D C N, G 6 P D$ and $L P L$ were all strongly anti-adipogenic in this study. It is possible that the effects of the two pro-adipogenic genes GPAM and DGAT2 on adipogenesis were not strong enough to lead the inhibitory of adipogenesis when targeted by the miR-23a 27a 24-2 cluster. Thus, we asked whether there were other pro-adipogenic target genes apart from GPAM and $D G A T 2$ that contribute to $m i R-23 a \sim 27 a \sim 24-2$ cluster-regulated preadipocyte differentiation. If this is true, the predominant pathway should be the pro-adipogenic (dotted green lines) pathway. 
A
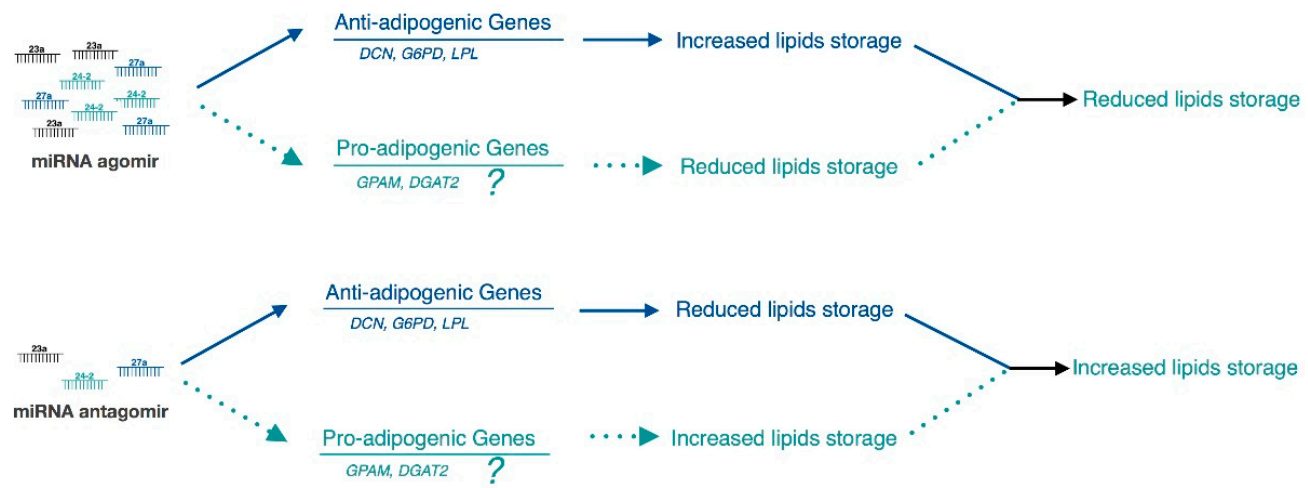

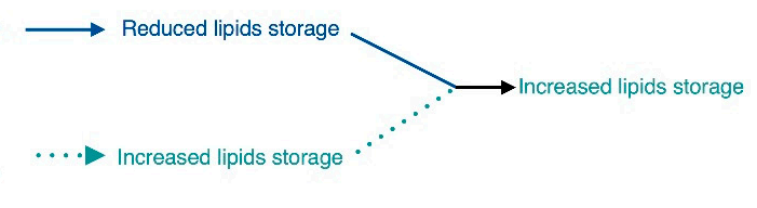

Figure 6. A hypothesis of the miR-23a 27a 24-2 cluster regulatory role in bovine adipocyte adipogenesis. In this study, miRNA agomir repressed adipogenesis (A) and miRNA antagomir increased adipogenesis (B). Since most of the proved target genes $(D C N, G 6 P D$, and $L P L)$ were strong anti-adipogenic effects (solid blue lines), we hypothesize that there were other pro-adipogenic target genes apart from GPAM and DGAT2 that contribute to $m i R-23 a \sim 27 a \sim 24-2$ cluster-regulated preadipocyte differentiation (dotted green lines).

\subsection{FGF11 Is a Direct Target of the miR-23a 27a 24-2 Cluster}

To investigate whether there are other pro-adipogenic genes that are regulated by the miR-23a 27a 24-2 cluster, predicted targets were further analyzed using TargetScan software and miRanda. Among the thousands of predicted genes, we identified a new gene that might be commonly targeted by $m i R-23 a$, miR-27a, and miR-24-2: FGF11. FGF11 is an intracellular fibroblast growth factor and is a potential novel mediator of adipogenesis [32]. We found that expression of FGF11 was clearly induced during adipocyte adipogenesis (Figure 7A). Furthermore, its expression pattern was opposite to that of $m i R-27 a$ and $m i R-24-2$. In addition, expression of FGF11 was tightly controlled by the miR-23a 27a 24-2 cluster. Agomir of miR-23a, miR-27a, and miR-24-2 significantly repressed FGF11 expression (Figure 7B). Conversely, inhibition of miR-23a, miR-27a, and miR-24-2 strongly increased FGF11 expression in adipocytes from $36 \mathrm{~h}$ to 8 days post transfection with miRNA antagomir (Figure 7C-E). These results indicate that FGF11 is a potential target gene of the $m i R-23 a \sim 27 a \sim 24-2$ cluster.

A

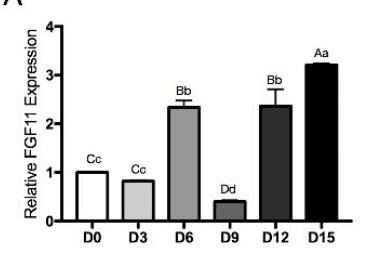

B

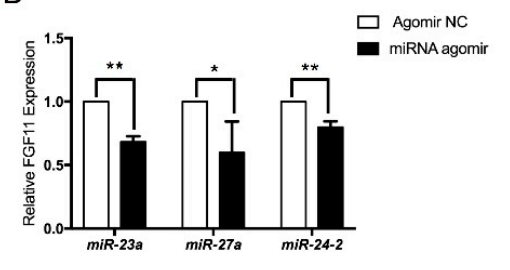

C

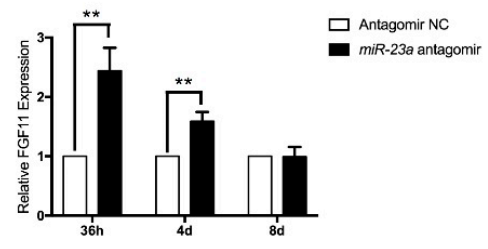

D

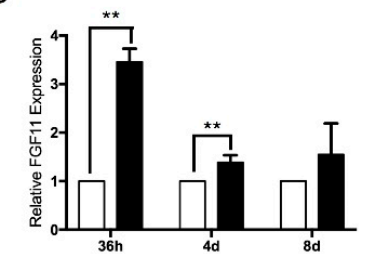

$\mathrm{E}$

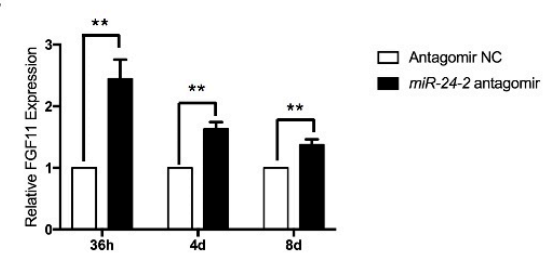

Figure 7. FGF11 expression is commonly regulated by $m i R-23 a, m i R-27 a$, and $m i R-24-2$. (A) Time course of FGF11 expression during preadipocyte differentiation period. (B) miR-23a agomir, miR-27a agomir, and miR-24-2 agomir suppressed FGF11 expression in adipocytes. (C-E) Transfection of miR-23a antagomir, miR-27a antagomir, and miR-24-2 antagomir increased FGF11 expression in differentiating adipocytes from $36 \mathrm{~h}$ to 8 days. Error bars represent SEM. Lowercase represents $p<0.05$. Uppercase represents $p<0.01$. “*” represents $p<0.05$. “**” represents $p<0.01$. 
To determine the relationship between the miR-23a 27a 24-2 cluster and FGF11, in vitro luciferase activity assays were performed in $293 \mathrm{~T}$ cells. Two potential binding sites were predicted within the 3'UTR of FGF11 mRNA (Figure 8A). The miR-24-2 seed sequence between the two binding sites was highly conserved among mammals (Figure 8B). Next the wild-type and mutant 3'UTRs containing the miRNA binding site seed sequence were chemically synthesized and cloned into the PsiCHECK ${ }^{\mathrm{TM}}-2$ vector (Figure 8C,D). Results showed that $m i R-24-2$ agomir significantly reduced luciferase activities of the FGF11 3'UTR reporter vector (Figure 8F) while miR-27a had no effects (Figure 8E). These results indicate that FGF11 is commonly regulated by $m i R-23 a, m i R-27 a$, and $m i R-24-2$, and is a direct target of $m i R-24-2$.

A
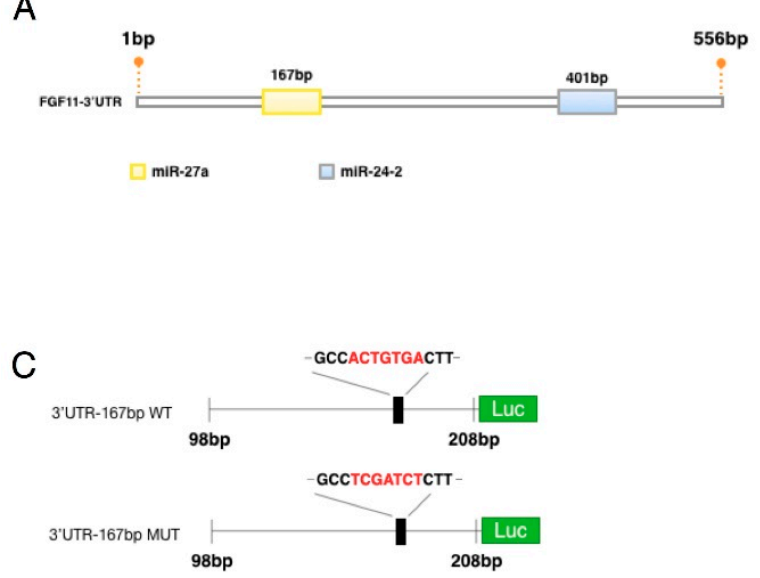

E

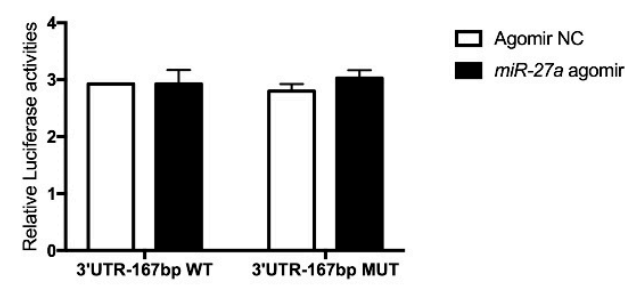

B

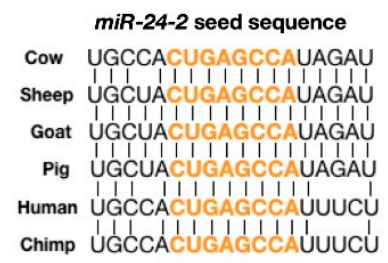

D

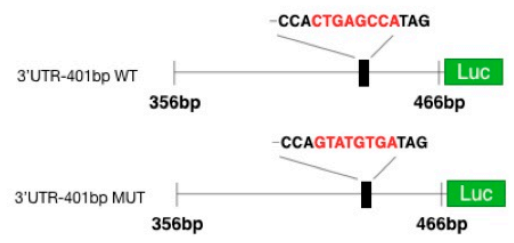

$\mathrm{F}$

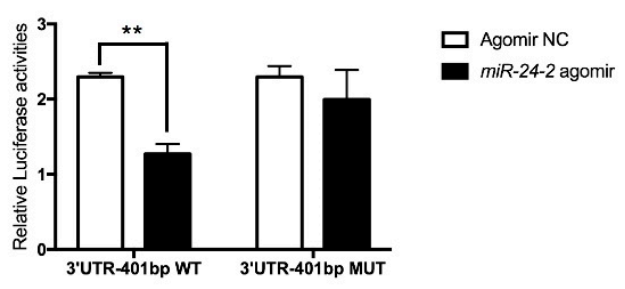

Figure 8. FGF11 is targeted by the miR-23a 27a 24-2 cluster. (A) Prediction of miR-23a, miR-27a, and miR-24-2 seed sequence in the FGF11 3'UTR region using TargetScan software and miRanda. (B) Sequence alignments of miR-24-2 seed sequence showed high conservation among mammals. (C,D) Structure of FGF11-3'UTR luciferase reporter vector with wild or mutant miRNA seed sequence. The mutant sequence was marked in red. (E,F) Luciferase analysis showed that $F G F 11$ was directly targeted by miR-24-2 (F) but not miR-27a (E). Error bars represent SEM. "***" represents $p<0.01$.

\subsection{FGF11 Is a Pro-Adipogenic Mediator of Adipogenesis}

An siRNA interference assay was employed to investigate the effects of FGF11 on adipocyte adipogenesis. Two FGF11 siRNA oligonucleotides were designed and transfected into preadipocytes to inhibit FGF11 expression. The siRNA with clearly higher interference efficiency $(82.7 \%)$, si-FGF11-401bp, was finally chosen for the interference assays (Figure 9A). We found that inhibition of FGF11 strongly reduced lipid droplet accumulation as visualized by Oil Red O staining (Figure 9B). ELISA analysis also showed that inhibition of FGF11 obviously reduced cytoplasm TG content (Figure 9C). These results indicate that FGF11 is a downstream pro-adipogenic target of the miR-23a 27a 24-2 cluster. The co-regulation of FGF11, GPAM, and DGAT2 might explain why preadipocyte differentiation is negatively regulated by the $m i R-23 a \sim 27 a \sim 24-2$ cluster. 
A

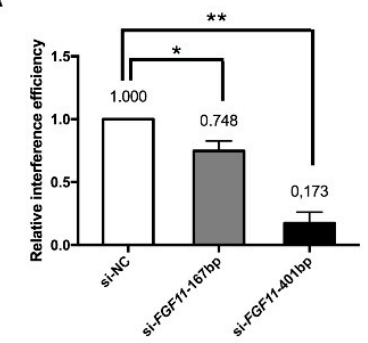

B

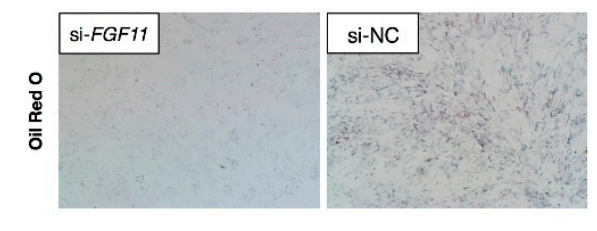

C

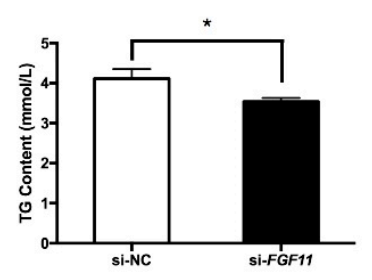

Figure 9. FGF11 is a pro-adipogenic mediator of adipogenesis. (A) Interference efficiency of siRNA targeting FGF11 mRNA in adipocytes. (B) Oil Red O staining of lipids in FGF11 interfered differentiating adipocytes (magnification: $40 \times$ ). (C) ELISA analysis of TG content in adipocytes when interfering FGF11 expression by siRNA. Error bars represent SEM. "*” represents $p<0.05$. “**” represents $p<0.01$.

\section{Discussion}

In beef cattle, fat deposition is closely associated with the meat quality of beef, which is one of the most important economic traits of beef production [33,34]. During adipogenesis, miRNA has been shown to be important for post-transcriptional regulation [35]. A previous study has demonstrated that in bovine subcutaneous adipose tissue, there are hundreds of miRNAs detectable with 17 miRNAs that are tissue-specifically expressed that might be involved in the regulation of energy balance [36]. Recently, it was reported that the predicted common targets of the miR-23a 27a 24-2 cluster are enriched in energy metabolism-related pathways [12]. However, to our knowledge, the cooperative and independent functions of the $m i R-23 a \sim 27 a \sim 24-2$ cluster in bovine adipocyte adipogenesis has not been examined.

Our study is the first to identify the cooperative functions of the miRNAs in the miR-23a 27a 24-2 cluster in adipogenesis. Our principal findings are: (1) the miRNAs in the miR-23a 27a 24-2 cluster inhibits bovine adipocyte adipogenesis; (2) the miRNAs in the miR-23a 27a 24-2 cluster individually regulate ATP5F1, DCN, GPAM, DGAT2, G6PD, and LPL and cooperatively regulate FGF11; and (3) the inhibitory effect on adipogenesis is achieved through the balanced regulation by targeting two types of genes with pro- or anti-adipogenic effects.

Several studies have reported the roles of $m i R-23 a$ and $m i R-27 a$ in preadipocyte differentiation. miR-23a was identified as a negative regulator of preadipocyte differentiation by Huang et al. [18] and Guan et al. [19]. Many studies have demonstrated that miR-27a negatively regulates adipogenesis by targeting PPARG, the key transcription factor in adipocyte terminal differentiation $[20,23,37,38]$. In this study we confirmed these previously reported roles of $m i R-23 a$ and miR-27a. We also showed that $m i R-24-2$ is a negative regulator of adipogenesis. Although both Huang et al. [18] and Guan et al. [19] measured miRNA expression during preadipocyte differentiation, conflicting expression patterns were reported. Huang et al. found that miR-23a expression first increased then decreased from day 2 to day 8 during 3T3-L1 preadipocyte differentiation [18]. However, Guan et al. reported that miR-23a was immediately down regulated during adipogenic differentiation of platelet derived growth factor receptor alpha (PDGFR $\alpha$ )-positive progenitor cells [19]. The expression pattern of miR-23a in our study was the same as that reported by Huang et al. As studies have reported that depot-specific differences in adipose tissue contribute differently to metabolism $[1,29,39]$, we think that these differences in expression patterns are understandable and are largely due to the different cell models used.

Traditionally, miRNA is considered to post-transcriptionally repress expression by binding to the 3'UTR of mRNA [40,41]. In most cases, when the relationship between an individual miRNA and its target gene is studied, the miRNA and its target gene are found to have opposite effects [42-44]. However, because a single miRNA might bind to multiple mRNAs and multiple miRNAs might regulate a single mRNA [12], it is possible that these targets have opposite effects, even in the same biological process. Thus, regulation of these genes may be a way to maintain homeostasis. Interestingly, 
in this study, we identified three direct target genes $(D C N, G 6 P D$, and $L P L)$ that have the same effects as what the miRNAs in the miR-23a 27a 24-2 cluster do on adipocyte adipogenesis and three that have the opposite effects (GPAM, DGAT2, and FGF11).

We found that miR-23a targets $D C N, m i R-24-2$ targets G6PD and $L P L$, and that all these three genes inhibit adipogenesis. DCN is a recently identified secreted protein that inhibits preadipocyte differentiation [29]. Considering that it binds to a variety of collagens, it is likely that $D C N$ inhibits adipogenesis through collagen-dependent pathways [45,46]. G6PD is a rate-limiting enzyme in the pentose phosphate pathway and is indispensable in de novo lipogenesis [24]. It has also been implicated in adipose tissue inflammation and systemic insulin resistance in obesity [27]. It was recently demonstrated that abnormal activation of G6PD increases cellular nicotinamide adenine dinucleotide phosphate (NADPH), resulting in lipid dysregulation in obesity [47]. However, here we found that G6PD clearly had the opposite effect on preadipocyte differentiation. This may be related to the locations of the fat depots where the cells were isolated [1]. LPL is a rate-limiting enzyme catalyzing the hydrolysis of TG and is synthesized and secreted by a variety of cell types including myocytes, adipocytes and mammary gland cells, implying that miR-24-2 could inhibit adipocyte adipogenesis by repressing the hydrolysis of TG by $L P L[30,48]$.

In the present study, we also identified three pro-adipogenic targets of the miR-23a 27a 24-2 cluster: GPAM was targeted by miR-27a, DGAT2 was targeted by miR-24-2, and FGF11 was commonly regulated by $m i R-23 a, m i R-27 a$, and $m i R-24-2$. GPAM, also known as glycerol 3-phosphate acyltransferase 1 (GPAT1), is one of four identified GPATs [49]. GPATs catalyze the first step of TAG synthesis and are rate-limiting enzymes in the de novo pathway of glycerolipid synthesis [50]. Studies have reported that mitochondrial GPAM greatly limits glycerolipid synthesis in bovine embryonic fibroblast cells [51]. Meanwhile, elevated GPAM expression level is related to higher bovine milk fat synthesis and increased adipose tissue lipogenesis [52,53]. DGAT2 is responsible for the final step of TAG synthesis: the conversion of diacylglycerols (DAGs) to TAG [54]. Thus, DGAT2 might strongly contribute to $m i R-23 a \sim 27 a \sim 24-2$ cluster-regulated inhibition of adipogenesis. We also found that FGF11 was commonly regulated via the miR-23a 27a 24-2 cluster. FGF11 is an intracellular fibroblast growth factor that is involved in angiogenesis, tumorigenesis, and liver regeneration [55]. Importantly, a microarray study found that FGF11 expression was dramatically increased during in vitro differentiation of human adipose-derived stem cells, indicating that FGF11 might be a novel mediator of adipogenesis [32]. Consistent with this, we identified that FGF11 is a positive regulator of preadipocyte differentiation. The contribution of the pro-adipogenic targets GPAM, DGAT2, and FGF11 to adipogenesis might be greater than that of DCN, G6PD, and LPL, and this may lead to the reduced adipogenesis phenotype observed in the presence of the miR-23a 27a 24-2 cluster. Although much more work is needed to elucidate the mechanisms underlying the cooperative functions of the miR-23a 27a 24-2 cluster in adipogenesis, the newly identified co-regulatory roles in this study will also help inform future studies on adipogenesis.

In the studies of animal breeding, genomic selection is a powerful approach to improve the quality of economic traits [56]. The pivotal issue for genomic selection is to establish the reference population and candidate population for better selection of the paternal and maternal candidates [57]. To improve the meat quality of beef, increasing the marbling (or intramuscular fat) content is an efficient and necessary approach [58]. Understanding the molecular basis of the miR-23a 27a 24-2 cluster in adipogenesis can provide new insights for selecting candidates with more intramuscular fat content. Consequently, the miR-23a 27a 24-2 cluster can be considered as a candidate target for genomic selection breeding in beef cattle to improve the meat quality.

In conclusion, the miR-23a 27a 24-2 cluster represses adipogenic differentiation of bovine preadipocytes. This inhibitory effect is achieved through a balanced regulation via targeting two types of genes with anti-adipogenic effects $(D C N, G 6 P D$, and $L P L)$ and pro-adipogenic effects (GPAM, DGAT2, and FGF11), which finally leads to the miR-23a 27a 24-2 cluster-mediated repression of adipogenesis (Figure 10). Even though this study did not reveal the detailed mechanisms underlying 
the regulation of the miR-23a 27a 24-2 cluster on adipogenesis, the newly identified cooperative and independent regulatory roles for the miRNAs in this cluster will provide insights into the mechanisms that govern how adipogenesis is regulated, and this knowledge can be used to increase the meat quality of beef.

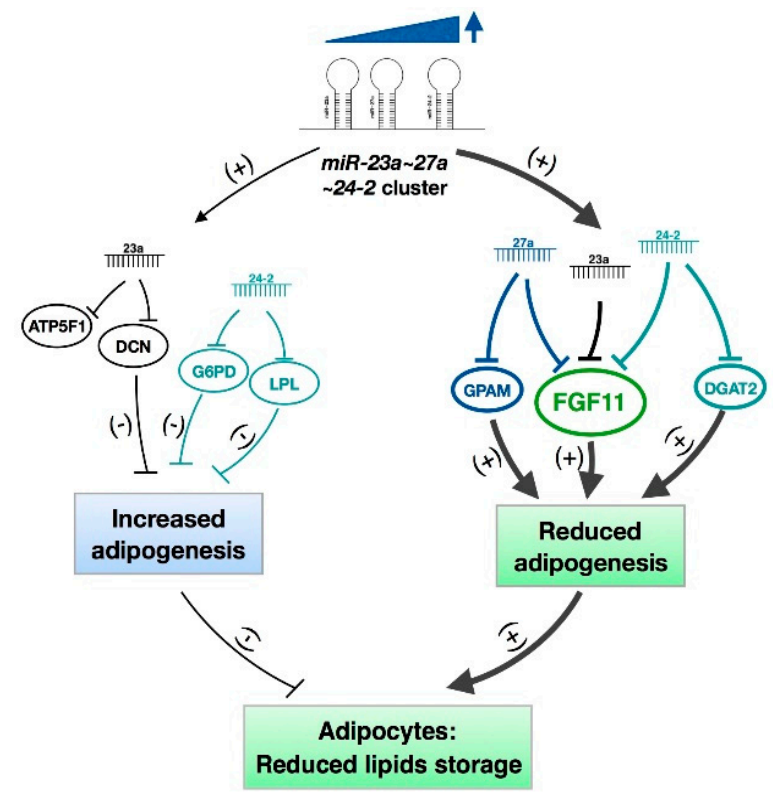

Figure 10. Mechanisms of the miR-23a 27a 24-2 cluster in regulating bovine adipocyte adipogenesis. The miR-23a 27a 24-2 cluster represses adipogenic differentiation of bovine preadipocytes. This inhibitory effect is achieved through a balanced regulation via targeting two types of genes with anti-adipogenic effects ( $D C N, G 6 P D$, and $L P L)$ and pro-adipogenic effects (GPAM, DGAT2, and FGF11), which finally leads to the $m i R-23 a \sim 27 a \sim 24-2$ cluster-mediated repression of adipogenesis.

\section{Materials and Methods}

\subsection{Isolation of Bovine Preadipocytes and Cell Culture}

Isolation and cell culture of preadipocytes were performed as previously described by Meissburger et al. and Wang et al. [29,59]. The experiments were performed in accordance with the guiding principles for the experimental animals adopted by Ministry of Science and Technology. Animal care was approved by the Institutional Animal Care and Use Committee of Northwest A\&F University (approved code: No.142, 2005). The adipose sample was obtained from a slaughter house. The perirenal adipose tissue was removed, placed into phosphate buffer saline (PBS) supplemented with $10 \%$ penicillin/streptomycin (Hyclone, ThermoFisher Scientific, Shanghai, China) and immediately taken to the cell culture lab. The blood vessels and connective tissues were carefully dissected away from the adipose tissue. The adipose sample was then minced and digested with $0.25 \%$ collagenase I (Sigma, Shanghai, China) for $1 \mathrm{~h}$ at $37^{\circ} \mathrm{C}$ in a shaking water bath. The cell suspension was filtered through a $40-\mu \mathrm{m}$ cell strainer and centrifuged for $5 \mathrm{~min}$ at $350 \times \mathrm{g}$ to obtain cell pellets. The pellets were resuspended and seeded in $60-\mathrm{mm}$ collagen-coated cell culture plates. The preadipocytes were grown in growth medium containing Dulbecco's Modified Eagle Medium/F-12 (DMEM/F-12, Gibco, Shanghai, China) supplemented with 10\% fetal bovine serum (FBS, Gibco) and $1 \%$ penicillin/streptomycin (Gibco). Growth medium was changed every two days and cells were passaged at $70 \%$ confluence to avoid spontaneous differentiation. For adipogenic differentiation, post-confluent cells were cultured in induction medium containing DMEM/F-12 supplemented with 10\% FBS, $1 \%$ penicillin/streptomycin, $5 \mu \mathrm{g} / \mathrm{mL}$ insulin (Sigma-Aldrich, Shanghai, China), $1 \mu \mathrm{M}$ dexamethasone (Sigma-Aldrich) and 0.5 mM 3-isobutyl-1-methylxanthine (IBMX, Sigma-Aldrich) 
for 2 days. Then, every 2 days the cells were incubated in fresh maintenance medium containing DMEM/F-12 supplemented with 10\% FBS, 1\% penicillin/streptomycin, and $5 \mu \mathrm{g} / \mathrm{mL}$ insulin.

\subsection{Oil Red O Staining}

Lipid droplets were visualized using Oil Red O staining [60]. The Oil Red O stock solution was prepared by dissolving $0.5 \mathrm{~g}$ powder in isopropanol, and the solution was protected from light. The working solution was prepared by diluting the stock solution to $60 \%$ with sterile deionized water. To stain lipids, cells were washed with PBS, and fixed with $4 \%$ paraformaldehyde for $30 \mathrm{~min}$ at room temperature. After that, cells were rinsed with PBS three times and stained with Oil Red O working solution for $30 \mathrm{~min}$ at room temperature. The fluorescent dye 4',6-diamidino-2-phenylindole (DAPI) was used to stain the cell nuclei at a concentration of $1.43 \mu \mathrm{M}$. Bright field and fluorescent microscopy were taken with an inverted fluorescence microscope (Olympus IX71, Olympus Corporation, Beijing, China).

\subsection{RNA Oligonucleotides and Transfection}

Chemically modified agomir and antagomir were used to increase and inhibit, respectively, the expression of $m i R-23 a, m i R-27 a$, and $m i R-24-2$. The miRNA agomir and antagomir were synthesized and purified using high-performance liquid chromatography by GenePharma Co. Ltd (Shanghai, China). The sequences of the miRNA oligonucleotides are listed in Tables S1 and S2. The short interference RNA (siRNA) oligonucleotides targeting DCN, GPAM, DGAT2, G6PD, and LPL mRNAs and the negative control (NC) siRNA were designed using the online BLOCK-iT ${ }^{\mathrm{TM}}$ RNAi Designer (http://rnaidesigner.thermofisher.com/rnaiexpress). The siRNA oligonucleotides were chemically synthesized without modifications and purified using high-performance liquid chromatography by Sangon Biotech Co. Ltd (Shanghai, China). The sequences of the siRNA oligonucleotides are listed in Table S3.

For transfection of RNA oligonucleotides, cells were seeded in six-well cell culture plates in triplicate for each treatment. When the cells had grown to 70\% confluence, miRNA agomir or siRNA oligonucleotides were transfected into cells using lipofectamine ${ }^{\mathrm{TM}} 3000$ transfection reagent (Invitrogen, Shanghai, China) at a final concentration of $30 \mathrm{nM}$. miRNA antagomir was transfected at a final concentration of $75 \mathrm{nM}$ according to the manufacturer's protocol. Briefly, RNA oligonucleotides and transfection reagent were separately diluted in Opti-MEM medium (Gibco) and incubated for $10 \mathrm{~min}$ at room temperature. Next, the two mixtures were combined and incubated for another $15 \mathrm{~min}$ at room temperature to allow for the formation of transfection reagent-RNA complexes. The transfection complexes were then added to the cell culture medium drop by drop. Cells were incubated for $24 \mathrm{~h}$ before changing to a fresh growth medium.

\subsection{Luciferase Activity Assay}

To construct the luciferase reporter vector, $\approx 100-200 \mathrm{bp}$ of the bovine $3^{\prime}$ untranslated region $\left(3^{\prime} \mathrm{UTR}\right)$ region containing the wild type or mutant miRNA binding site was chemically synthesized (Sangon Biotech Co., Ltd.) and cloned into the psiCHECK ${ }^{\mathrm{TM}}-2$ (Promega, Beijing, China) vector. For co-transfection of miRNA oligonucleotides and the vector, 293A cells were seeded in 24-well cell culture plates in triplicate. For each treatment, $100 \mu \mathrm{g}$ plasmid and $50 \mathrm{nM}$ miRNA oligonucleotides were mixed and transfected into cells. The transfection protocol was the same as that used for miRNA and siRNA transfection.

The luciferase activity was measured $40 \mathrm{~h}$ after transfection using the Dual-Luciferase Reporter Assay System (Promega). In brief, cells were lysed in 1× Passive Lysis Buffer for $15 \mathrm{~min}$ at room temperature. To measure the firefly luciferase activity, $25 \mu \mathrm{L}$ Luciferase Assay Buffer II was mixed with $10 \mu \mathrm{L}$ cell lysate, and the absorbance was measured at $560 \mathrm{~nm}$ (TECAN, Infinite1 200 PRO NanoQuant, BioTek, Beijing, China). The Renilla luciferase activity was determined by adding $50 \mu \mathrm{L} 1 \times$ Stop 
\& Glo1 reagent to the cell lysate above and the absorbance was measured at $480 \mathrm{~nm}$. The relative luciferase activity was calculated as the ratio of Renilla luciferase activity to firefly luciferase activity.

\subsection{ELISA Analysis of Bovine Triglyceride Content}

The TG content of the adipocytes was measured using the Bovine TG ELISA Kit (Jining Shiye, Shanghai, China). Briefly, equal number of cells were scraped into an equal volume of PBS and were repeatedly frozen and thawed to release the TG within the adipocyte cytoplasm. Cells were then centrifuged for $25 \mathrm{~min}$ at $2500 \mathrm{rpm}$ to obtain the supernatant. The samples were then processed according to the manufacturer's instructions. The final absorbance was measured at $450 \mathrm{~nm}$ (TECAN, Infinite1 200 PRO NanoQuant).

\subsection{Quantitative Real-Time-PCR}

Total RNA from the adipocytes $(n=3)$ was isolated using the RNAiso Plus kit (Takara Biomedical Technology Co., Ltd., Beijing, China) according to the manufacturer's instructions. RNA was then reversely transcribed into cDNA using the Prime-Script ${ }^{\mathrm{TM}}$ RT reagent Kit with gDNA Eraser (Takara Biomedical Technology Co., Ltd.) for mRNA or the miRcute Plus miRNA First-Strand cDNA Synthesis Kit (TIANGEN, Beijing, China) for miRNA. The cDNA was then used as a template for qRT-PCR in triplicate wells in the 7500 Real Time PCR System (Applied Biosystems, Shanghai, China). TB Green ${ }^{\mathrm{TM}}$ Premix Ex Taq ${ }^{\mathrm{TM}}$ II (Tli RNaseH Plus, Takara, Beijing, China) was used for protein-coding gene analysis and the miRcute Plus miRNA qPCR Detection Kit (SYBR Green, TIANGEN) was used for miRNA analysis. The mRNA expression level was normalized to that of glyceraldehyde-3-phosphate dehydrogenase $(G A P D H)$ and the miRNA expression level was normalized to that of U6. Three repeats of the qRT-PCR assays were performed. The experimental data were analyzed using the $2^{-\Delta \Delta C t}$ method [61]. Sequence information for the primers are provided in Table S4.

\subsection{Statistical Analysis}

All data were presented as the mean \pm SEM. Statistically significant differences between two groups were tested using the independent-samples $t$-test, and significances among three or more groups were tested using one-way analysis of variance (ANOVA) [43].

Supplementary Materials: Supplementary materials can be found at http:/ / www.mdpi.com/1422-0067/19/12/ $3957 /$ s1.

Author Contributions: Y.W. designed experiments, performed experiments, analyzed data, and wrote the manuscript. Y.Z. performed essential experiments and contributed funding. X.S. and H.W. provided essential ideas. W.Y. provided essential reagents. L.Z. made manuscript revisions and contributed funding.

Funding: This research was funded by the National Natural Science Foundation of China (Grant No. 31501937), the National Key Research and Development Program of China (No. 2018YFD0501700), the National 863 Program of China (No.2013AA102505), Collaborative Innovation Major Projects of Yangling Demonstration Zone (No. 2017CXY-14), and the National Science and Technology Support Projects (No. 2015BAD03B04).

Acknowledgments: We would like to thank all of our collaborators, research assistants, and laboratory technicians who contributed to the study.

Conflicts of Interest: The authors declare no conflict of interest.

\section{Abbreviations}

GPAM glycerol-3-phosphate acyltransferase: mitochondrial

DGAT2 diacylglycerol O-acyltransferase 2

DCN decorin

G6PD glucose-6-phosphate dehydrogenase

LPL lipoprotein lipase

FGF11 fibroblast growth factor 11

FBS fetal bovine serum 


$\begin{array}{ll}\text { IBMX } & \text { 3-isobutyl-1-methylxanthine } \\ \text { SiRNA } & \text { short interference RNA } \\ \text { NC } & \text { negative control } \\ 3^{\prime} \text { UTR } & 3^{\prime} \text { untranslated region } \\ \text { TG } & \text { triglyceride } \\ \text { qRT-PCR } & \text { quantitative real time-PCR } \\ \text { ANOVA } & \text { one-way analysis of variance } \\ \text { ATP5F1 } & \text { ATP synthase peripheral stalk-membrane subunit b } \\ \text { TAG } & \text { triacylglycerol }\end{array}$

\section{References}

1. Rosen, E.D.; Spiegelman, B.M. What we talk about when we talk about fat. Cell 2014, 156, 20-44. [CrossRef]

2. Rosen, E.D.; MacDougald, O.A. Adipocyte differentiation from the inside out. Nat. Rev. Mol. Cell Biol. 2006, 7,885-896. [CrossRef]

3. Cristancho, A.G.; Lazar, M.A. Forming functional fat: A growing understanding of adipocyte differentiation. Nat. Rev. Mol. Cell Biol. 2011, 12, 722-734. [CrossRef] [PubMed]

4. Hilton, C.; Neville, M.J.; Karpe, F. MicroRNAs in adipose tissue: Their role in adipogenesis and obesity. Int. J. Obes. 2012, 37, 325-332. [CrossRef]

5. Arner, P.; Kulyte, A. MicroRNA regulatory networks in human adipose tissue and obesity. Nat. Rev. Endocrinol. 2015, 11, 276-288. [CrossRef] [PubMed]

6. Ha, M.; Kim, V.N. Regulation of microRNA biogenesis. Nat. Rev. Mol. Cell Biol. 2014, 15, 509-524. [CrossRef] [PubMed]

7. Mourelatos, Z.; Dostie, J.; Paushkin, S.; Sharma, A.; Charroux, B.; Abel, L.; Rappsilber, J.; Mann, M.; Dreyfuss, G. miRNPs: A novel class of ribonucleoproteins containing numerous microRNAs. Gene. Dev. 2002, 16, 720-728. [CrossRef]

8. Seitz, H.; Royo, H.; Bortolin, M.L.; Lin, S.P.; Ferguson-Smith, A.C.; Cavaille, J. A large imprinted microRNA gene cluster at the mouse Dlk1-Gt12 domain. Genome Res. 2004, 14, 1741-1748. [CrossRef]

9. Griffiths-Jones, S.; Saini, K.H.; van Dongen, S.; Enright, A.J. miRBase: Tools for microRNA genomics. Nucleic Acids. Res. 2008, 36, D154-158. [CrossRef] [PubMed]

10. Lee, Y.; Kim, M.; Han, J.; Yeom, K.H.; Lee, S.; Baek, S.H.; Kim, V.N. MicroRNA genes are transcribed by RNA polymerase II. EMBO J. 2004, 23, 4051-4060. [CrossRef] [PubMed]

11. Altuvia, Y.; Landgraf, P.; Lithwick, G.; Elefant, N.; Pfeffer, S.; Aravin, A.; Brownstein, M.J.; Tuschl, T.; Margalit, H. Clustering and conservation patterns of human microRNAs. Nucleic Acids. Res. 2005, 33, 2697-2706. [CrossRef] [PubMed]

12. Chhabra, R.; Dubey, R.; Saini, N. Cooperative and individualistic functions of the microRNAs in the miR-23a 27a 24-2 cluster and its implication in human diseases. Mol. Cancer 2010, 9, 232. [CrossRef] [PubMed]

13. Heider, T.; Mutschelknaus, L.; Radulovic, V.; Winkler, K.; Kimmel, J.; Anastasov, N.; Atkinson, M.J.; Moertl, S. Radiation induced transcriptional and post-transcriptional regulation of the hsa-miR-23a 27a 24-2 cluster suppresses apoptosis by stabilizing XIAP. BBA-Gene Regul. Mech. 2017, 1860, 1127-1137. [CrossRef] [PubMed]

14. Hassan, M.Q.; Gordon, J.A.; Beloti, M.M.; Croce, C.M.; van Wijnen, A.J.; Stein, J.L.; Stein, G.S.; Lian, J.B. A network connecting Runx2, SATB2, and the miR-23a 27a 24-2 cluster regulates the osteoblast differentiation program. Proc. Natl. Acad. SCI. USA 2010, 107, 19879-19884. [CrossRef] [PubMed]

15. Zeng, H.; Bae, Y.; Dawson, B.C.; Chen, Y.; Bertin, T.; Munivez, E.; Campeau, P.M.; Tao, J.; Chen, R.; Lee, B.H. MicroRNA miR-23a cluster promotes osteocyte differentiation by regulating TGF-beta signalling in osteoblasts. Nat. Commun. 2017, 8, 15000. [CrossRef] [PubMed]

16. Chen, Q.; Xu, J.; Li, L.; Li, H.; Mao, S.; Zhang, F.; Zen, K.; Zhang, C.Y.; Zhang, Q. MicroRNA-23a/b and microRNA-27a/b suppress Apaf-1 protein and alleviate hypoxia-induced neuronal apoptosis. Cell Death. Dis. 2014, 5, e1132. [CrossRef]

17. Wang, F.; Zhu, Y.; Guo, L.; Dong, L.; Liu, H.; Yin, H.; Zhang, Z.; Li, Y.; Liu, C.; Ma, Y.; et al. A regulatory circuit comprising GATA1/2 switch and microRNA-27a/24 promotes erythropoiesis. Nucleic Acids. Res. 2014, 42, 442-457. [CrossRef] 
18. Huang, Y.; Huang, J.; Qi, R.; Wang, Q.; Wu, Y.; Wang, J. Effects of MicroRNA-23a on Differentiation and Gene Expression Profiles in 3T3-L1 Adipocytes. Genes-Basel 2016, 7, 92. [CrossRef]

19. Guan, L.; Hu, X.; Liu, L.; Xing, Y.; Zhou, Z.; Liang, X.; Yang, Q.; Jin, S.; Bao, J.; Gao, H. bta-miR-23a involves in adipogenesis of progenitor cells derived from fetal bovine skeletal muscle. Sci. Rep. 2017, 7, 43716. [CrossRef]

20. Zou, B.; Ge, Z.; Zhu, W.; Xu, Z.; Li, C. Persimmon tannin represses 3T3-L1 preadipocyte differentiation via up-regulating expression of miR-27 and down-regulating expression of peroxisome proliferator-activated receptor-gamma in the early phase of adipogenesis. Eur. J. Nutr. 2015, 54, 1333-4133. [CrossRef]

21. Sun, L.; Trajkovski, M. MiR-27 orchestrates the transcriptional regulation of brown adipogenesis. Metabolism 2014, 63, 272-282. [CrossRef] [PubMed]

22. Wang, T.; Li, M.; Guan, J.; Li, P.; Wang, H.; Guo, Y.; Shuai, S.; Li, X. MicroRNAs miR-27a and miR-143 regulate porcine adipocyte lipid metabolism. Int. J. Mol. Sci. 2011, 12, 7950-7959. [CrossRef] [PubMed]

23. Yao, F.; Yu, Y.; Feng, L.; Li, J.; Zhang, M.; Lan, X.; Yan, X.; Liu, Y.; Guan, F.; Zhang, M. Adipogenic miR-27a in adipose tissue upregulates macrophage activation via inhibiting PPARgamma of insulin resistance induced by high-fat diet-associated obesity. Exp. Cell Res. 2017, 355, 105-112. [CrossRef] [PubMed]

24. Ham, M.; Choe, S.S.; Shin, K.C.; Choi, G.; Kim, J.W.; Noh, J.R.; Kim, Y.H.; Ryu, J.W.; Yoon, K.H.; Lee, C.H.; et al. Glucose-6-Phosphate Dehydrogenase Deficiency Improves Insulin Resistance With Reduced Adipose Tissue Inflammation in Obesity. Diabetes 2016, 65, 2624-2638. [CrossRef] [PubMed]

25. Fan, Q.; Long, B.; Yan, G.; Wang, Z.; Shi, M.; Bao, X.; Hu, J.; Li, X.; Chen, C.; Zheng, Z.; et al. Dietary leucine supplementation alters energy metabolism and induces slow-to-fast transitions in longissimus dorsi muscle of weanling piglets. Brit. J. Nutr. 2017, 117, 1222-1234. [CrossRef] [PubMed]

26. Bhatt-Wessel, B.; Jordan, T.W.; Miller, J.H.; Peng, L. Role of DGAT enzymes in triacylglycerol metabolism. Arch. Biochem. Biophys. 2018, 655, 1-11. [CrossRef] [PubMed]

27. Park, Y.J.; Choe, S.S.; Sohn, J.H.; Kim, J.B. The role of glucose-6-phosphate dehydrogenase in adipose tissue inflammation in obesity. Adipocyte 2017, 6, 147-153. [CrossRef]

28. Morgan-Bathke, M.; Chen, L.; Oberschneider, E.; Harteneck, D.; Jensen, M.D. More insights into a human adipose tissue GPAT activity assay. Adipocyte 2015, 5, 93-96. [CrossRef]

29. Meissburger, B.; Perdikari, A.; Moest, H.; Muller, S.; Geiger, M.; Wolfrum, C. Regulation of adipogenesis by paracrine factors from adipose stromal-vascular fraction-A link to fat depot-specific differences. BBA-Mol. Cell Biol. L. 2016, 1861, 1121-1131. [CrossRef]

30. He, P.; Jiang, T.; OuYang, X.; Liang, Y.; Zou, J.; Wang, Y.; Shen, Q.; Liao, L.; Zheng, X. Lipoprotein lipase: Biosynthesis, regulatory factors, and its role in atherosclerosis and other diseases. Clin. Chim. Acta 2018, 480, 126-137. [CrossRef]

31. Clark, A.L.; Naya, F.J. MicroRNAs in the Myocyte Enhancer Factor 2 (MEF2)-regulatedGt12-Dio3Noncoding RNA Locus Promote Cardiomyocyte Proliferation by Targeting the Transcriptional Coactivator Cited2. J. Biol. Chem. 2015, 290, 23162-23172. [CrossRef] [PubMed]

32. Satish, L.; Krill-Burger, J.M.; Gallo, P.H.; Etages, S.D.; Liu, F.; Philips, B.J.; Ravuri, S.; Marra, K.G.; LaFramboise, W.A.; Kathju, S. Expression analysis of human adipose-derived stem cells during in vitro differentiation to an adipocyte lineage. BMC Med. Genom. 2015, 8, 41. [CrossRef] [PubMed]

33. Ottaviani, E.; Malagoli, D.; Franceschi, C. The evolution of the adipose tissue: A neglected enigma. Gen. Comp. Endocr. 2011, 174, 1-4. [CrossRef] [PubMed]

34. Zhang, Y.; Wang, H.; Wang, Y.; Wang, H.; Zhang, S.; Hong, J.; Guo, H.; Chen, D.; Yang, Y.; Zan, L. Transcriptome analysis of mRNA and microRNAs in intramuscular fat tissues of castrated and intact male Chinese Qinchuan cattle. PLoS ONE 2017, 12, e0185961. [CrossRef] [PubMed]

35. Romao, J.M.; Jin, W.; Dodson, M.V.; Hausman, G.J.; Moore, S.S.; Guan, L.L. MicroRNA regulation in mammalian adipogenesis. Exp. Biol. Med. 2011, 236, 997-1004. [CrossRef] [PubMed]

36. Romao, J.M.; Jin, W.W.; He, M.L.; McAllister, T.; Guan, L.L. MicroRNAs in bovine adipogenesis: Genomic context, expression and function. BMC Genom. 2014, 15, 137. [CrossRef] [PubMed]

37. Lin, Q.; Gao, Z.; Alarcon, R.M.; Ye, J.; Yun, Z. A role of miR-27 in the regulation of adipogenesis. FEBS J. 2009, 276, 2348-2358. [CrossRef]

38. Kim, S.Y.; Kim, A.Y.; Lee, H.W.; Son, Y.H.; Lee, G.Y.; Lee, J.W.; Lee, Y.S.; Kim, J.B. miR-27a is a negative regulator of adipocyte differentiation via suppressing PPARgamma expression. Biochem. Biophys. Res. Commun. 2010, 392, 323-328. [CrossRef] 
39. Joe, A.W.; Yi, L.; Even, Y.; Vogl, A.W.; Rossi, F.M. Depot-specific differences in adipogenic progenitor abundance and proliferative response to high-fat diet. Stem Cells 2009, 27, 2563-2570. [CrossRef]

40. Filipowicz, W.; Bhattacharyya, S.N.; Sonenberg, N. Mechanisms of post-transcriptional regulation by microRNAs: Are the answers in sight? Nat. Rev. Genet. 2008, 9, 102-114. [CrossRef]

41. Gebert, L.F.R.; MacRae, I.J. Regulation of microRNA function in animals. Nat. Rev. Mol. Cell Biol. 2018. [CrossRef]

42. Wang, Q.; Li, Y.C.; Wang, J.; Kong, J.; Qi, Y.; Quigg, R.J.; Li, X. miR-17-92 cluster accelerates adipocyte differentiation by negatively regulating tumor-suppressor Rb2/p130. Proc. Natl. Acad. SCI. USA 2008, 105, 2889-2894. [CrossRef] [PubMed]

43. Snyder, C.M.; Rice, A.L.; Estrella, N.L.; Held, A.; Kandarian, S.C.; Naya, F.J. MEF2A regulates the Gtl2-Dio3 microRNA mega-cluster to modulate WNT signaling in skeletal muscle regeneration. Development 2012, 140, 31-42. [CrossRef] [PubMed]

44. Wust, S.; Drose, S.; Heidler, J.; Wittig, I.; Klockner, I.; Franko, A.; Bonke, E.; Gunther, S.; Gartner, U.; Boettger, T.; et al. Metabolic Maturation during Muscle Stem Cell Differentiation Is Achieved by miR-1/133a-Mediated Inhibition of the Dlk1-Dio3 Mega Gene Cluster. Cell Metab. 2018, 27, 1026-1039. [CrossRef] [PubMed]

45. Ibrahimi, A.; Bonino, F.; Bardon, S.; Ailhaud, G.; Dani, C. Essential Role of Collagens for Terminal Differentiation of Preadipocytes. Biochem. Biophys. Res. Commun. 1992, 187, 1314-1322. [CrossRef]

46. Wiberg, C.; Heinegard, D.; Wenglen, C.; Timpl, R.; Morgelin, M. Biglycan organizes collagen VI into hexagonal-like networks resembling tissue structures. J. Biol. Chem. 2002, 277, 49120-49126. [CrossRef]

47. Koh, H.J.; Lee, S.M.; Son, B.G.; Lee, S.H.; Ryoo, Z.Y.; Chang, K.T.; Park, J.W.; Park, D.C.; Song, B.J.; Veech, R.L.; et al. Cytosolic NADP+-dependent isocitrate dehydrogenase plays a key role in lipid metabolism. J. Biol. Chem. 2004, 279, 39968-39974. [CrossRef]

48. Dijk, W.; Ruppert, P.M.M.; Oost, L.J.; Kersten, S. Angiopoietin-like 4 promotes the intracellular cleavage of lipoprotein lipase by PCSK3/furin in adipocytes. J. Biol. Chem. 2018, 293, 14134-14145. [CrossRef]

49. Gonzalez-Baro, M.R.; Lewin, T.M.; Coleman, R.A. Coleman, Regulation of Triglyceride Metabolism. II. Function of mitochondrial GPAT1 in the regulation of triacylglycerol biosynthesis and insulin action. Am. J. Phys.-Gastrointest. Liver 2007, 292, G1195-G1199. [CrossRef]

50. Yu, J.; Loh, K.; Song, Z.; Yang, H.; Zhang, Y.; Lin, S. Update on glycerol-3-phosphate acyltransferases: The roles in the development of insulin resistance. Nutr. Diabetes 2018, 8, 34. [CrossRef]

51. Yu, H.; Zhao, Z.; Yu, X.; Li, J.; Lu, C.; Yang, R. Bovine lipid metabolism related gene GPAM: Molecular characterization, function identification, and association analysis with fat deposition traits. Gene 2017, 609, 9-18. [CrossRef] [PubMed]

52. Bionaz, M.; Loor, J.J. Gene networks driving bovine milk fat synthesis during the lactation cycle. BMC Genom. 2008, 9, 366. [CrossRef] [PubMed]

53. Crookenden, M.A.; Mandok, K.S.; Grala, T.M.; Phyn, C.V.; Kay, J.K.; Greenwood, S.L.; Roche, J.R. Source of metabolizable energy affects gene transcription in metabolic pathways in adipose and liver tissue of nonlactating, pregnant dairy cows. J. Anim. Sci. 2015, 93, 685-698. [CrossRef] [PubMed]

54. Meegalla, R.L.; Billheimer, J.T.; Cheng, D. Concerted elevation of acyl-coenzyme A: Diacylglycerol acyltransferase (DGAT) activity through independent stimulation of mRNA expression of DGAT1 and DGAT2 by carbohydrate and insulin. Biochem. Biophys. Res. Commun. 2002, 298, 317-323. [CrossRef]

55. Lee, K.W.; Yim, H.S.; Shin, J.; Lee, C.; Lee, J.H.; Jeong, J.Y. FGF11 induced by hypoxia interacts with HIF-1alpha and enhances its stability. FEBS Lett. 2017, 591, 348-357. [CrossRef] [PubMed]

56. Wiggans, G.R.; Cole, J.B.; Hubbard, S.M.; Sonstegard, T.S. Genomic Selection in Dairy Cattle: The USDA Experience. Annu. Rev. Anim. Biosci. 2017, 5, 309-327. [CrossRef] [PubMed]

57. Rabier, C.E.; Barre, P.; Asp, T.; Charmet, G.; Mangin, B. On the Accuracy of Genomic Selection. PLoS ONE 2016, 11, e0156086. [CrossRef]

58. Galvez, F.; Maggiolino, A.; Dominguez, R.; Pateiro, M.; Gil, S.; De Palo, P.; Carballo, J.; Franco, D.; Lorenzo, J.M. Nutritional and meat quality characteristics of seven primal cuts from 9-month old female veal calves: A preliminary study. J. Sci. Food Agric. 2018. [CrossRef]

59. Wang, Y.; Yang, W.; Li, P.; Wang, H.; Zhang, Y.; Zan, L. Myocyte enhancer factor 2A promotes proliferation and its inhibition attenuates myogenic differentiation via myozenin 2 in bovine skeletal muscle myoblast. PLoS ONE 2018, 13, e0196255. [CrossRef] 
60. Lee, Y.H.; Kim, S.H.; Lee, Y.J.; Kang, E.S.; Lee, B.W.; Cha, B.S.; Kim, J.W.; Song, D.H.; Lee, H.C. Transcription factor Snail is a novel regulator of adipocyte differentiation via inhibiting the expression of peroxisome proliferator-activated receptor gamma. Cell Mol. Life Sci. 2013, 70, 3959-3971. [CrossRef]

61. Liu, S.; Sun, G.; Yuan, B.; Zhang, L.; Gao, Y.; Jiang, H.; Dai, L.; Zhang, J. miR-375 negatively regulates porcine preadipocyte differentiation by targeting BMPR2. FEBS Lett. 2016, 590, 1417-1427. [CrossRef] [PubMed] 